\title{
Comportement migratoire du saumon Loire-Allier : le problème des obstacles
}

\author{
Migratory behaviour of Loire-Allier salmon : \\ the problems relating to obstacles
}

\author{
R. Cuinat
}

Conseil supérieur de la pêche

Délégation régionale Auvergne-Limousin

Études réalisées avec l'aide financière du Ministère de l'Environnement

Sur l'axe Loire-Allier, grande distance et multiplicité des obstacles entre mer et frayères ont "façonné " une souche de saumons atlantiques très particulière : entrée en estuaire précoce, forte proportion de sujets de grande taille, diversité des comportements aboutissant à "partager les risques". Le problème des obstacles et de leur influence est plus particulièrement discuté.

At the Loire-Allier axis the vast distance and multiplicity of problems between the sea and spawning places have resulted into a very special stock of Atlantic salmon being generated : access into precocious estuary, a considerable number of large fish, diversity of behaviour resulting in "sharing of risks ». The discussions are more particularly centred around the problems relating to obstacles and their impact.

Sur le bassin de la Loire, les parties de rivières susceptibles de convenir à la reproduction du Saumon, puis à sa vie juvénile, sont en quasi-totalité séparées de la mer par plusieurs centaines de kilomètres - 800 à 900 pour l'Allier. Cette donnée géographique majeure conditionne la biologie et le comportement de ce Saumon atlantique, au point d'avoir façonné une souche que l'on pouvait appeler "ligérienne "; nous disons maintenant "LoireAllier », puisque l'espèce ne se reproduit plus actuellement que dans ce dernier cours d'eau (fig. 1).

Nous rappellerons brièvement les diverses étapes du cycle vital de ces saumons, en examinant plus particuliè- rement les diverses stratégies de croissance et de migration qu'ils peuvent utiliser. Nous réfléchirons ensuite aux effets directs et indirects des obstacles sur le niveau de population sur l'axe Loire-Allier.

Sur un axe fluvial de cette dimension, les observations ou les données chiffrées sont difficiles, approximatives ou biaisées. Cet article sera donc considéré comme contestable sur plusieurs points. Mais, dans ce domaine, si nous attendons toujours d'avoir des certitudes pour donner des avis ou pour agir, nous risquons fort d'arriver trop tard : le Saumon aura disparu. 


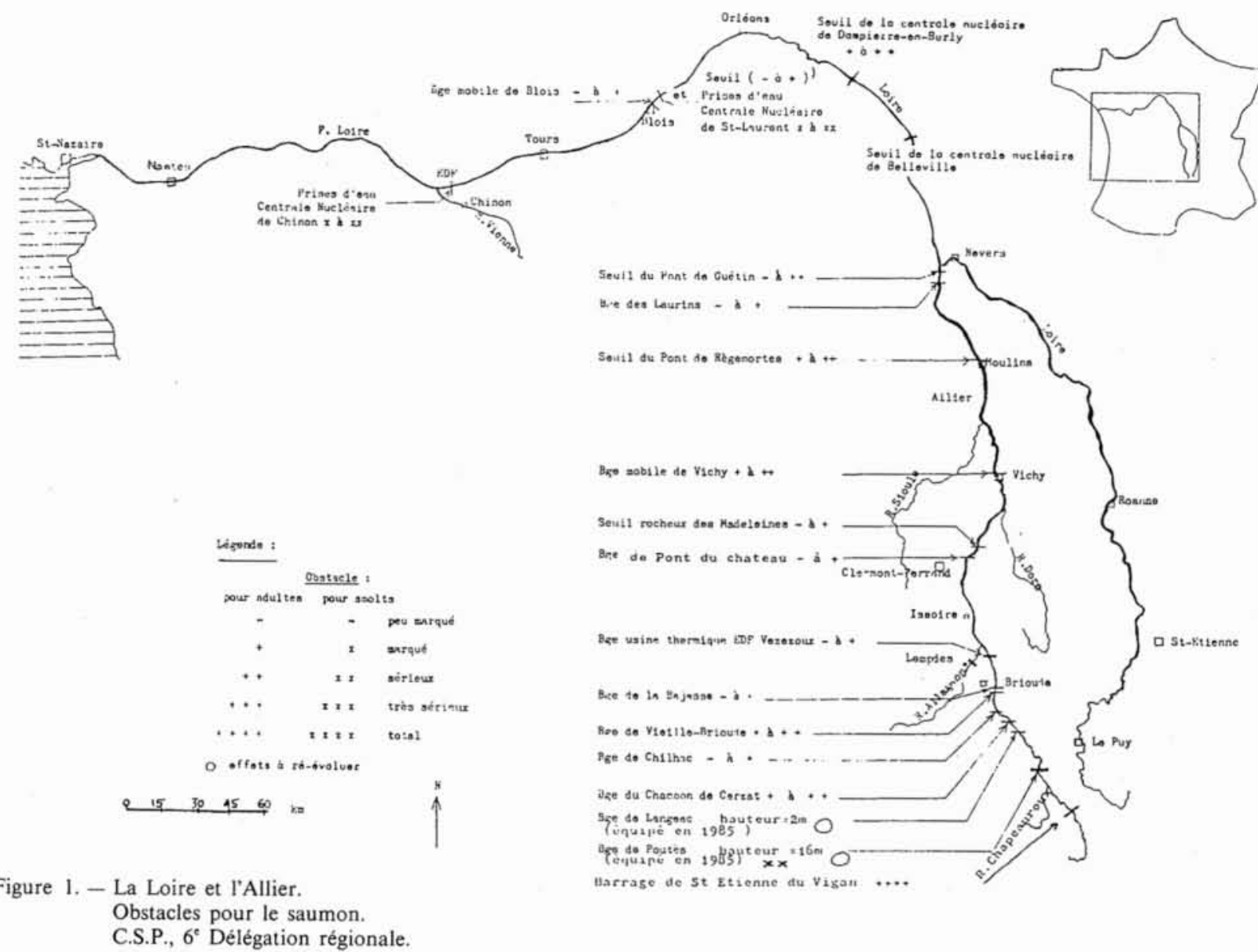

\section{Cycle vital. Diverses stratégies migratoires} (Cuinat, 1980 et Cuinat et Bomassi, 1985)

\section{A - Reproduction - Vie des juvéniles en rivière}

La reproduction commence au début novembre et peut durer jusqu'à la mi-décembre.

Seules conviennent à cette reproduction les eaux claires et rapides, sur fond de galets ou graviers, suffisamment meuble pour que la femelle puisse y creuser son nid (les parties de l'Allier situées en Haute-Loire sont les plus favorables; en aval, les fonds sont généralement plus colmatés, notamment à l'aval de Clermont-Ferrand, du fait de la pollution), par des battements rapides de sa nageoire caudale. Les ovules, fécondés par le mâle (donc devenus œufs), pénètrent entre les galets.

$\mathrm{Si}$ la frayère n'a pas été bouleversée par une crue ou colmatée par des limons, les œufs éclosent en fin d'hiver. Les alevins ne sortiront des graviers qu'au printemps, lorsque leur vésicule vitelline sera résorbée.

La croissance des jeunes saumons est rapide dans l'Allier. Au printemps suivant, les deux tiers environ des survivants atteignent 12 à $18 \mathrm{~cm}$, se "smoltifient " et dévalent vers la mer. Les autres attendront l'âge de deux ans, exceptionnellement trois. Ces «saumoneaux» (ou "smolts ") parcourent entre 10 et 30 kilomètres par jour.
Certains ouvrages, notamment Saint-Laurent-des-Eaux, provoquent des retards ou des mortalités sur une portion variable de la population (Cuinat et al., 1980). Ce problème n'est pas traité dans le présent article.

En mer, nous perdons la trace de ces jeunes poissons.

\section{B - Vie marine}

D'après les renvois de marques, les captures de saumons Loire-Allier en mer se font principalement à l'Ouest du Groënland. Nos poissons y côtoient leurs confrères canadiens; se nourrissant (comme les phoques) principalement de capelans, ils "s'engraissent " de deux kilos par an en moyenne.

La durée du séjour en mer, pour le Saumon LoireAllier, varie beaucoup selon les sujets : certains reviennent au bout d'un an et demi (c'est-à-dire après 2 étés passés en mer; il est possible même que certains reviennent après un an seulement de mer, mais échappent à nos échantillonnages du fait qu'ils arrivent en eau douce lorsque la pêche y est officiellement terminée), pesant quatre à cinq kilos; les autres ne le font qu'après deux ans et demi ou trois ans et demi (c'est-à-dire après 3 ou 4 étés en mer); ils pèsent alors cinq à dix kilos, parfois plus. La forte proportion de "grands saumons" est caractéristique de l'axe Loire-Allier, ainsi que de quelques autres grands 
cours d'eau de Scandinavie ou du Canada.

La navigation en mer, et notamment le retour à l'estuaire, s'appuie sur un instinct héréditaire, avec perception du champ magnétique terrestre et de la position des astres.

En eau douce, c'est l'olfaction qui jouera le rôle prépondérant : les adultes cherchent à retrouver " le goût de l'eau " qu'ils avaient mémorisé dans leur jeunesse, et tout particulièement à leur stade saumoneau, au cours de leur descente vers la mer.

\section{C - Remontée des adultes}

Arrivés en estuaire, les saumons se ré-habituent progressivement à l'eau douce et cessent de s'alimenter.

La période d'arrivée en estuaire est très variable selon les sujets :

a) Certains y rentrent dès le début de l'automne, c'est-à-dire plus d'un an avant la ponte; ce faisant, ils ont un maximum de chances de franchir successivement tous les obstacles et de parvenir en temps voulú aux bonnes zones de reproduction. En contre-partie, ils se privent de toute nourriture pendant une longue période de leur vie, dans laquelle leur croissance pondérale est négative. Ceci tout en restant exposés à des mortalités naturelles et par pêche.

b) D'autres y rentrent en hiver.

c) Enfin, d'autres n'arrivent qu'au printemps : à l'opposé du premier contingent, ils auront profité d'une croissance supplémentaire. Par contre, il est probable qu'ils ne parviendront à se reproduire que les années oủ les circonstances hydrologiques et climatiques permettent une migration assez rapide.

Il est évident que la multiplication et l'aggravation des obstacles réduit les chances des contingents tardifs, dont le « rendement » (survie $\times$ croissance) aurait pourtant été le meilleur. Sur la plupart des autres cours d'eau à saumons, en France et dans le Monde, les saumons ne quittent la mer qu'au printemps, parfois en été et même en automne, soit parce que les fleuves sont régulièrement pris en glace une partie de l'année, soit que quelques mois - ou semaines - leur suffisent pour parvenir aux zones de frai.

Notons aussi que les contingents précoces et les contingents tardifs sont généralement moins exposés à la pêche fluviale (aux filets en Loire, à la ligne dans l'Allier) : ils passent soit avant soit après la période d'ouverture de cette pêche (depuis une dizaine d'années, les dates d'ouverture - actuellement étagées de janvier à début mars d'aval en amont - ont été retardées de façon à épargner les sujets précoces. La fermeture est au 15 juin -31 juillet en 1986).

La vitesse de remontée de la Loire, puis de l'Allier, est extrêmement variable selon les sujets et selon les conditions hydrologiques et climatiques. Ainsi, les premiers saumons peuvent aborder la zone des bonnes frayères dès janvier certaines années particulièrement propices, ou seulement en avril ou mai en conditions défavorables. La montée est entrecoupée de périodes d'arrêt. Les zones de repos s'échelonnent tout au long de l'Allier, et sont plus ou moins fréquentées par les saumons selon les conditions de débit. C'est généralement là que les recherchent les pêcheurs à la ligne.
En été, les saumons, peu actifs, se retranchent dans des parties profondes. A l'occasion des crues (ou " coups d'eau ") de septembre à novembre, ils cherchent à se rapprocher des zones précises de frai.

Les femelles choisissent le plus souvent pour cela les "têtes de courant". Les saumons Loire-Allier cherchent-ils à pondre à l'endroit où ils sont nés ? Leur " homing " est indiscutable : très peu de sujets "s'égarent " vers des affluents précédemment non colonisés; mais la précision de ce retour est mal connue, et semble admettre une certaine dispersion, d'ailleurs favorable à l'extension de l'espèce.

Lors de la ponte, les saumons Loire-Allier sont très amaigris. Une très faible proportion survivra pour un deuxième voyage aller-retour et une reproduction ultérieure, contrairement à ce qui se passe sur des fleuves courts.

\section{D - Cycle du saumon Loire-Allier}

La vie juvénile en rivière pouvant durer un à trois ans, la phase marine deux (peut-être un) à quatre ans et la remontée fluviale six à quinze mois, la durée totale du cycle varie selon les sujets entre quatre (peut-être trois) ?) et sept ans, la majorité bouclant ce cycle en quatre (15\%), cinq $(55 \%)$ et six $(26 \%)$ années (pourcentages moyens, susceptibles de varier très sensiblement selon les cohortes).

Cette diversité des comportements, si elle complique les approches du biologiste, a probablement des conséquences utiles à l'espèce, notamment :

a) En cas d'absence totale de reproduction une année A (par exemple suite à un blocage accidentel de la migration, ou à un bouleversement des frayères par une forte crue), il n'y aura pas un vide complet l'année A + 1 chez les juvéniles, ni les années $\mathrm{A}+4, \mathrm{~A}+5$ ou $\mathrm{A}+$ 6 pour la reproduction.

b) Le mélange de plusieurs cohortes sur les lieux de reproduction réduit les risques de consanguinité.

c) Les " stratégies " de croissance-migration-reproduction sont diversifiées. Ceux des sujets restant plus de deux ans en mer subissent nécessairement plus de mortalités que les autres, mais sont peut-être mieux adaptés aux difficultés de montée sur l'axe Loire-Allier. Ils sont en tout cas plus prolifiques (une femelle pond en moyenne 1800 ovules par kilo de son poids : par contre, chez les mâles, le nombre de spermatozoïdes est de toutes façons excédentaire - plusieurs milliards par sujet; la taille influe donc moins sur la prolificité; justement, les mâles semblent généralement séjourner moins d'années en mer que les femelles).

\section{Effets des obstacles}

Sur l'axe Loire-Allier, il y a actuellement dix neuf " obstacles» (seuils ou barrages), soit cinq sur la Loire et quatorze sur l'Allier (incluant le barrage de SaintEtienne-du-Vigan, en Lozère). Mais ce nombre n'est qu'indicatif, le terme " obstacle " étant parfois très subjectif. 


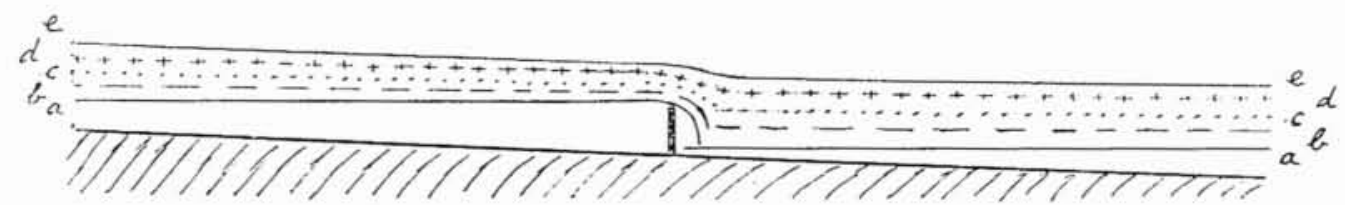

Figure 2. - Diminution de la hauteur de chûte au niveau d'un barrage, en fonction des débits (a, b, c, d, e) de la rivière (schéma) - En basses eaux (a), la chute est de deux mètres; en crue (e), elle est à peine visible.
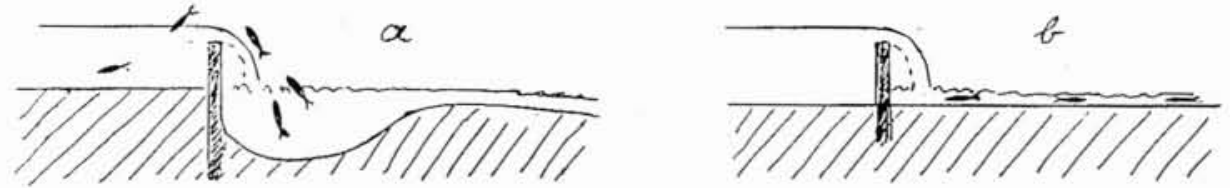

Figure 3. - Possibilité (a) ou non (b) de franchissement, pour une même hauteur de chute, selon la profondeur d'eau au pied de cette chute.
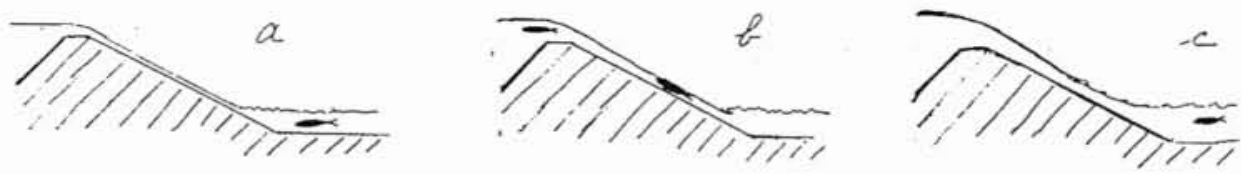

Figure 4. - Possibilité (b) ou non (a) de nage sur le parement aval incliné d'un barrage, selon le débit. a : lame d'eau trop mince; $b$ : lame d'eau suffisante; $c$ : lame d'eau trop rapide.

\section{A - Facteurs rendant un obstacle plus ou moins franchissable}

\section{1 - L'obstacle lui-même}

En gros, un obstacle est d'autant plus difficile à franchir qu'il occasionne une différence de niveau amont-aval plus importante. Mais cette différence diminue quand le débit de la rivière augmente (fig. 2 ), certains obstacles pouvant devenir peu visibles en crue. Pour la quasi-totalité des ouvrages sur la Loire et l'Allier, la hauteur de chute est en augmentation sensible (jusqu'à trois mètres à Moulins) depuis une vingtaine d'années, du fait des extractions de granulats et de la réduction du débit solide qui en est résulté. Depuis 1978-1980, ces extractions sont interdites dans la rivière Allier, sauf sa partie aval (Nièvre et Cher); elles restent autorisées en Loire.

Hauteur de chute, débit et configuration des lieux conditionnent les vitesses de l'eau, elles-mêmes très variables selon la proximité de la rive, la profondeur, etc. Lorsque les vitesses sont trop importantes pour leurs possibilités de nage, il reste aux saumons le saut. Mais celui-ci est difficile lorsque la hauteur d'eau est faible au pied de la chute. Ainsi, au petit barrage de Pont-duChâteau (63), une dénivellation de l'ordre d'un mètre seulement arrêtait souvent les saumons, la hauteur d'eau en aval n'étant que de quelques décimètres.

Le saut est inefficace pour une forte proportion de poissons lorsque le courant est rapide à l'amont immédiat de la chute : ceux-ci sont refoulés vers l'aval dès qu'ils retombent : cas notamment du radier fixe du Pont-Barrage de Vichy. Sur des barrages à parement aval non vertical (Brioude, Vieille-Brioude, Langeac), c'est sur ce parement et non dans le bief amont que les saumons retombent après leur saut; seule la nage est donc possible pour de tels obstacles; encore faut-il que les débits soient suffisants et que l'épaisseur de la lame d'eau permette cette nage (mais pas trop importants, pour que la vitesse de cette eau ne soit pas excessive !) La " plage de débits acceptables » est donc d'autant plus réduite que l'arasement de la crète du barrage est plus parfaite). De plus, sur de tels barrages, le " bourrelet " parfois ajouté à la crète arrête souvent les poissons qui avaient pu nager jusque là.

Pour la nage comme pour le saut, la quantité de bulles d'air brassées avec l'eau accroît généralement les difficultés, la densité du mélange étant réduite.

Dans une veine d'eau particulièrement rapide, il arrive parfois que certains saumons "déjaugent " et se propulsent par battements très rapides de leur nageoire caudale, le reste du corps étant presque entièrement hors de l'eau. 


\section{2 - Obstacles équipés de dispositifs de franchisse- ment}

Tous les obstacles sérieux sur l'Allier, excepté Pont-du-Guétin à l'aval et Saint-Etienne-du-Vigan à l'amont, sont maintenant équipés d'un ou plusieurs « dispositifs de franchissement " (y compris, depuis 1986, le barrage E.D.F. de Poutès-Monistrol, en Haute-Loire). Sur la Loire en aval du Bec d'Allier, plusieurs obstacles restent à équiper.

La question des passes à poissons est traitée dans d'autres documents. Nous rappellerons seulement ici que, dans beaucoup de cas sur l'Allier, malgré l'existence d'un dispositif de franchissement (ou même de plusieurs), les barrages ou seuils restent encore des obstacles:

a) Soit parce que les saumons semblent trouver difficilement ces passes. C'est souvent le cas pour les barrages construits perpendiculairement à l'axe du cours d'eau (cas de Vichy); toutefois ce problème est lié également à la direction et à la "puissance " des courants existant en aval du barrage : ainsi, à Moulins (radier perpendiculaire), les saumons trouvent généralement bien la passe, située en rive droite, grâce à la composante transversale créée par un épi rocheux au pied de l'obstacle.

b) Soit parce que les saumons sont moins attirés vers le barrage (et la passe) que par la restitution d'une usine hydro-électrique: cas de la microcentrale de VieilleBrioude, et probablement de Poutès-Monistrol.

\section{3 - Facteurs physiologiques}

La température de l'eau influence le comportement des saumons, mais nos observations sont encore bien peu précises à ce sujet :

La chaleur (plus de 16 à $18^{\circ} \mathrm{C}$ dans l'Allier) réduit "l'ardeur" des saumons à remonter la rivière, et à franchir les obstacles.

Le froid également: ainsi, au barrage de Vichy, on constate qu'en avril, si l'eau n'atteint pas 10 à $12^{\circ} \mathrm{C}$, les passages de saumons sont quasi-nuls dans les passes à poissons. Par contre, il semble qu'en novembre-décembre, ces passes soient fréquentées même à des températures plus basses. Sur la Loire, en fin d'hiver, les saumons n'essaient de franchir les seuils de Dampierre ou de Belleville que lorsque l'eau dépasse 5 à $6^{\circ} \mathrm{C}$ (d'après les radio-pistages réalisés par la Délégation régionale du C.S.P. de Poitiers en 1983,84 et 85 , avec l'aide financière d'E.D.F.

Ainsi, l'effet des températures sur l'activité migratoire est bien certain; les seuils sont toutefois différents selon la saison (ou peut-être selon "l'ancienneté " de l'arrivée en eau douce?).

Après l'été, la vigueur des saumons est généralement diminuée, quelles que soient les températures. Ceci pourrait provenir de leur amaigrissement, et du développement plus avancé de leurs gonades.

Dans les quelques passes à poissons sur l'Allier où nous avons pu contrôler les passages, on constate que ceux-ci s'arrêtent généralement la nuit, mais sont par contre assez intenses à l'aube, au crépuscule, ainsi semble-t-il que lorsque l'éclairement n'est pas trop intense. Mais ces renseignements resteraient à préciser.

Sur l'axe Loire-Allier comme sur la quasi-totalité des autres cours d'eau, on sait que les crues, ou simplement les "coups d'eau", déclenchent ou activent les activités migratoires. D'après nos observations fragmentaires, il semblerait que cette stimulation soit surtout liée aux augmentations naturelles, qui s'accompagnent probablement d'odeurs particulières liées à la pluie ou au ruissellement; les augmentations artificielles, provoquées par des lâchures ou turbinages à partir de retenues, ne semblent pas aussi efficaces; mais ceci reste à préciser davantage. Ceci semble valable également pour l'entrée en estuaire, bien que les observations y soient difficiles : les débits de la Loire sont restés exceptionnellement bas de l'été 1985 jusqu'à janvier 1986; il semble que les arrivées de saumons, en estuaire comme en basse-Loire, aient été à peu près nulles jusqu'en janvier 1986, alors qu'elles commencent habituellement en septembre ou octobre (mais il est possible aussi que des saumons soient entrés en estuaire et y soient morts du fait de la pollution, très accrue par cette sécheresse).

Parmi les autres facteurs pouvant influencer la montée, il faut citer la pêche. D'après Thioulouze (1979), la concentration de pêcheurs lançant des leurres lourds, ou le combat d'un saumon piqué à l'hameçon, ou surtout l'odeur du sang d'un sujet blessé, déclenchent un comportement de fuite vers l'aval ou d'arrêt de la montée.

Il est probable aussi que les "filets-barrages ", tradionnellement utilisés sur la Loire, ont eux aussi un effet retardateur, surtout s'ils occupent une grande partie de la largeur du cours d'eau.

D'autres facteurs de stress contrarient la montée, notamment, semble-t-il, les manipulations que nous avons pratiquées certaines années pour transporter des saumons à l'amont d'obstacles particulièrement difficiles, les extractions de granulats dans le lit du cours d'eau, etc. La campagne de radio-pistage entreprise en 1986 sur l'Allier confirme cette observation: une partie des saumons capturés, endormis, puis relâchés après ingestion forcée d'un micro-émetteur, sont retournés presque aussitôt vers l'aval, jusqu'à cinquante kilomètres.

Il est probable que la pollution de l'eau perturbe le comportement migratoire et les performances, mais du fait qu'elle est généralement associée à des périodes de faibles débits, nous avons peu de données précises à ce sujet.

\section{B - Comportement des saumons au niveau d'un obstacle}

Parfois, on observe directement les sauts de saumons tentant, avec succès ou en vain, de franchir l'obstacle. Dans certains cas, comme à Vichy à certaines périodes, il est clair qu'une grande partie des saumons ne trouvent pas les échelles à poissons. Ils dépensent alors certainement beaucoup d'énergie à se maintenir dans les eaux rapides ou émulsionnées au pied de l'obstacle.

Mais bien souvent, on n'observe presque rien, sinon une augmentation rapide de la concentration de pêcheurs en aval, du moins là où la pêche est autorisée. Les « zones d'attente " des saumons peuvent s'étendre sur plusieurs kilomètres sur l'Allier, les poissons séjournant plutôt dans des parties assez profondes. Ont-ils perçu l'obstacle de loin? Sont-ils avertis par des congénères? Attendent-ils d'être entraînés par certains d'entre eux, jouant le rôle de " chef de file " (Thioulouze, 1979) ? Sont-ils allés jusqu'au pied de l'obstacle, ont-ils tenté de le franchir, et ont-ils 
rebroussé chemin, attendant des circonstances plus favorables ? Aucune réponse précise n'est actuellement possible (1).

Dans certains cas, l'obstacle provoque aussi une concentration de saumons à l'amont immédiat, sur quelques centaines de mètres, comme si ces poissons se reposaient après l'effort; des réserves, généralement courtes, ont donc été mises en place à l'amont de la plupart des obstacles sur l'Allier.

Le comportement des saumons dans les passes à poissons " observables" est variable selon les sujets. Ces poissons font quelquefois demi-tour, surtout s'ils sont dérangés. Parfois, ils s'arrêtent longtemps juste avant la sortie de l'échelle, sans motif apparent pour nous, et peuvent même redescendre ensuite.

\section{C - Obstacles absolus et obstacles relatifs}

Les paragraphes précédents nous font comprendre qu'entre l'obstacle absolu, ou "total permanent" (un cas seulement sur l'axe Loire-Allier : Saint-Etienne-du-Vigan, hauteur $\simeq 15 \mathrm{~m}$ ), et « l'obstacle entièrement neutralisé ", il y a tous les intermédiaires.

Sur l'Allier ", mis à part les barrages de Pont-duChâteau (63) et de Brioude ("La Bajasse ", 43), tous les seuils ou barrages semblent provoquer, dans certaines conditions, des ralentissements et des concentrations de saumons, avec des fréquences évidemment différentes selon les sites (le barrage de Vichy étant celui qui pose le plus souvent des problèmes).

Ceci explique l'ampleur des polémiques autour de ces ouvrages. Monsieur X qui a vu cinq saumons sauter et retomber en aval, dira: "Le barrage de A est infranchissable " (il ajoutera même peut-être. "Il y a des centaines de saumons bloqués dessous ", alors que c'est peut-être le même poisson qui a sauté cinq fois). Mais Monsieur Y, qui est venu trois semaines plus tard, au moment d'un coup d'eau, et a vu passer cinq saumons, dira: "Au barrage de $\mathrm{A}$, les saumons passent facilement ». Quant à Monsieur Z, pêcheur qui habite à proximité, il ne se plaint pas de ce que des saumons s'arrêtent là de temps en temps, et ne voit pas l'utilité d'améliorer les possibilités de franchissement. Pour des raisons financières, le Maître d'Ouvrage partage généralement son point de vue.

\section{D - Effets des obstacles relatifs}

A première vue, un obstacle n'est pas très dangereux s'il suffit que les saumons attendent l'arrivée de circonstances favorables pour le franchir. L'expérience montre qu'il n'en est rien.

(1) Depuis 1973-1974, pour éviter les captures excessives de saumons dans ces zones, des réserves de pêche ont été instituées sur des distances de 0,5 à 1,5 kilomètres à l'aval de tous les obstacles de la rivière Allier.

En aval des obstacles particulièrement difficiles, comme Vichy, la longueur de la réserve devrait à notre avis être augmentée. En Loire, la longueur des réserves instituées à l'aval des obstacles n'atteint généralement pas un kilomètre; elle devrait également être augmentée, si l'on voulait favoriser l'accroissement du cheptel pour les prochaines années.

\section{1 - L'attente à l'aval a de multiples conséquences nuisibles au cheptel:}

a) Elle provoque des concentrations anormales de poissons, vite repérées par les pêcheurs, et permettant des captures souvent inespérées. La disparition progressive du Saumon de tous les affluents de l'axe Loire-Allier, ainsi que de la Loire supérieure, est riche d'enseignements à cet égard (Bachelier 1964): Chaque fois qu'un barrage a été édifié ou surélevé, les populations locales, alliées en cela au responsable de l'ouvrage, n'ont pas soutenu celles de l'amont qui souhaitaient que l'on remédie à la situation. Lorsque, après plusieurs années, la raréfaction du cheptel devint évidente, il fut souvent trop tard pour agir; d'autant plus qu'entre temps, d'autres barrages s'étaient parfois édifiés ou surélevés en amont, avec d'autant moins de précautions qu'il restait moins de saumons, et que chacun chargeait les autres de toutes les responsabilités.

b) Les chances de parvenir aux obstacles relatifs, situés en amont, dans des circonstances favorables sont diminuées. A titre d'exemple: La microcentrale de Vieille-Brioude n'est généralement bien franchissable que par débits assez importants, observés le plus fréquemment avant le mois de mai. Si, par suite d'eaux froides en avril, une partie des saumons a été retardée de quelques semaines à Vichy, Vieille-Brioude risque de devenir pour eux infranchissable; ils seront alors contraints de se reproduire dans des parties de l'Allier moins favorables. L'effet cumulé de plusieurs obstacles relatifs peut être l'interdiction totale de parvenir jusqu'aux frayères, pour une partie plus ou moins grande du contingent; la fraction la plus touchée sera souvent celle rentrée tardivement en estuaire, dont nous avons vu au chapitre I qu'elle avait par ailleurs le meilleur " rendement ".

2 - L'énergie dépensée par les saumons pour rechercher un passage, ou tenter de franchir l'obstacle, ne se récupérera pas ensuite, puisque les saumons ne s'alimentent plus. La fatigue accumulé réduira les chances d'une partie d'entre eux de franchir les obstacles suivants, et même de survivre jusqu'à la reproduction.

3 - Les obstacles relatifs sont pernicieux, du fait qu'ils laissent place aux controverses (sont-ils ou non " franchissables " ? Les échelles sont-elles ou non " efficaces " ?), qui retardent les solutions. La réalité est beaucoup plus complexe et fluctuante. Le fait que l'on ait constaté la réussite de saumons particulièrement vigoureux ne signifie pas que les autres aient pu passer. En réalité, c'est à l'échelle de la population dans son ensemble, et du parcours entier, de l'estuaire jusqu'aux frayères, que la question doit être traitée.

\section{Conclusion}

La population de saumons Loire-Allier se caractérise, par rapport à celles d'autres cours d'eau français, par un cycle de vie souvent long et des adultes généralement de grande taille, mais aussi par une très large diversité des comportements migratoires. Ces caractères sont probablement adaptés aux très grandes distances à parcourir, aux obstacles à franchir, et aux importantes fluctuations climatiques possibles selon les périodes et selon les années. 
DIAGRAMHES SCHEMATIQUES DE ROUTE D.UNE MONTEE DE SAUMONS RENCONTRANT DES OBSTACLES "RELATIFS", C'est a diro franchisgables uniquement lors de crues ( $C 1, C 2$, C3), ot par des saumons ( $A$ a $H$ ) en bonneg conditions (avant l'été).

a) Malgró lo retard pris au niveau des obstacles li et III, tous les saumons

(traits pleing) parviendront en mai dans la zone convenant a la reproduction. Si un obstacle relatif supplémentairo (I) est créb, les poissons (traits interrompus) $n$ 'y parviendront pas, sauf crue (improbable) en juin.

b) La présence d'obatacles relatifs concentro log flux migratoires.

c) Co diagramme suppose des poissons ayant tous 10 meme comportemnt migratoire. Il ne prend pas en compte : - la fatique augmentéo par les difficultés de franchissement des obstacles ;

- L'accroissement des mortalités par peche, du fait do la concentration a l'aval des obstacles.

Ainsi, meme "franchisgables", les obstacles "relatifs" aboutissent a róduire le nombre de reproducteurg survivant en fin de saison, ainsi que leurs chances de parvenir dans la zone des frayeres.

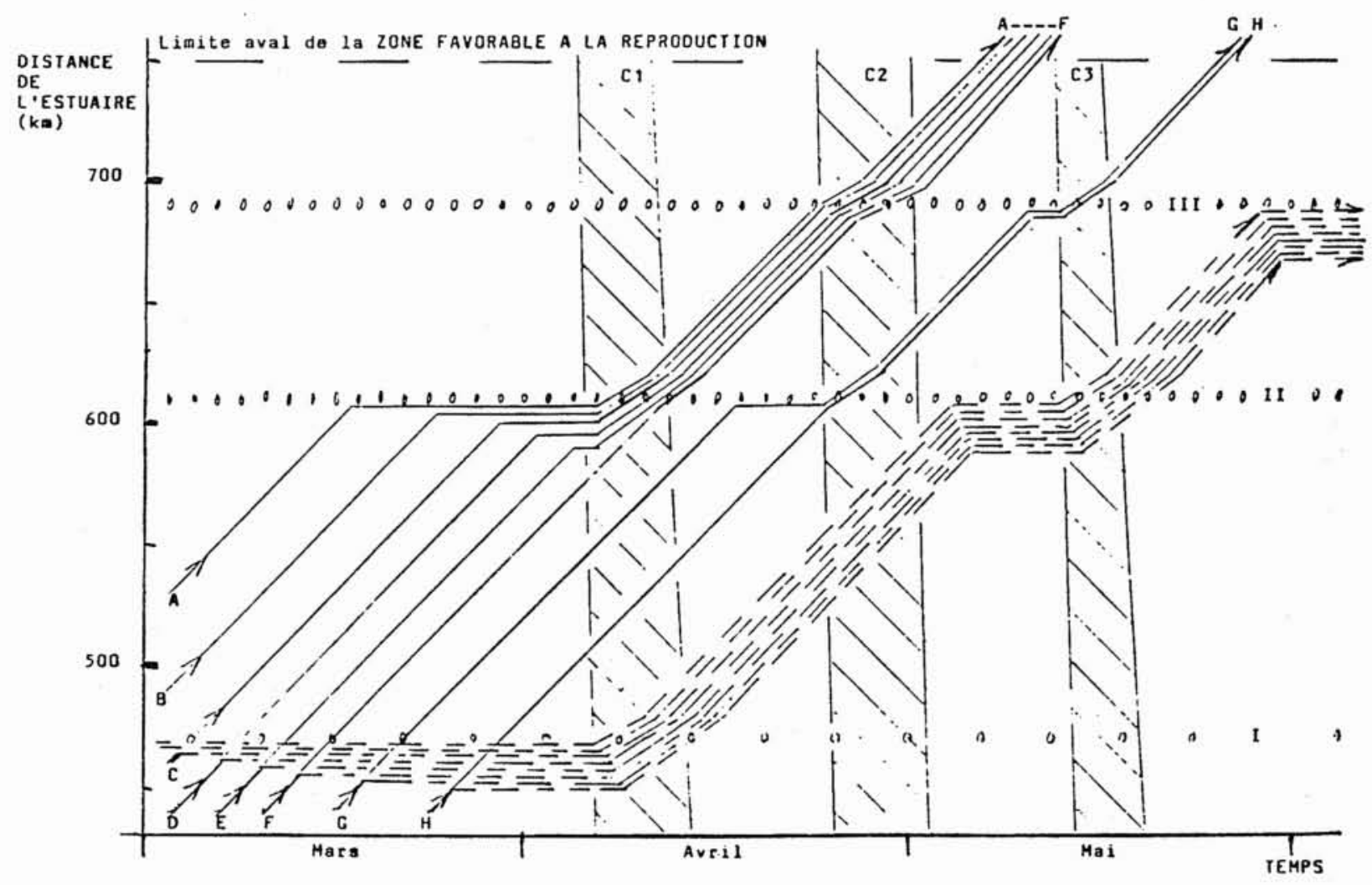

Figure 5 
DIAGRAMHES SCHEMATIQUES DE HIGRATION DE SAUMONS ADULTES, DE L'ESTUAIRE
LOIRE AUX FRAYERES ALLIER.

Flux do saumons

- P1 "prbcoces", dens une annbe où les conditione de eigration en eau douce sont favorablos (forts debits, tempratures pas trop basses)

- Pz "prócoces*, dens une anné a conditions dofavorables

+ II "tardifs", annbe favorablo

$x \quad 12$ "tardifa", anne defavorable

La densite des points diainue on fonction des mortalites (principaleaent par peche)

Zones tros favorables la reproduction: tb (tb : = zane en voie de recolonisation, du 8 arrage do Poutbs celui do St-Etienno-du-Viqan)

Zonea favorables : b

Zones atdiocres =

Les saumons précoces survivants parviendront aux zones de reproduction ( $t b$, b), atmo si les conditions de aigration sont dofavorables. Les tardifs n'y arriveront que los annosa ou los conditions sont favorables.

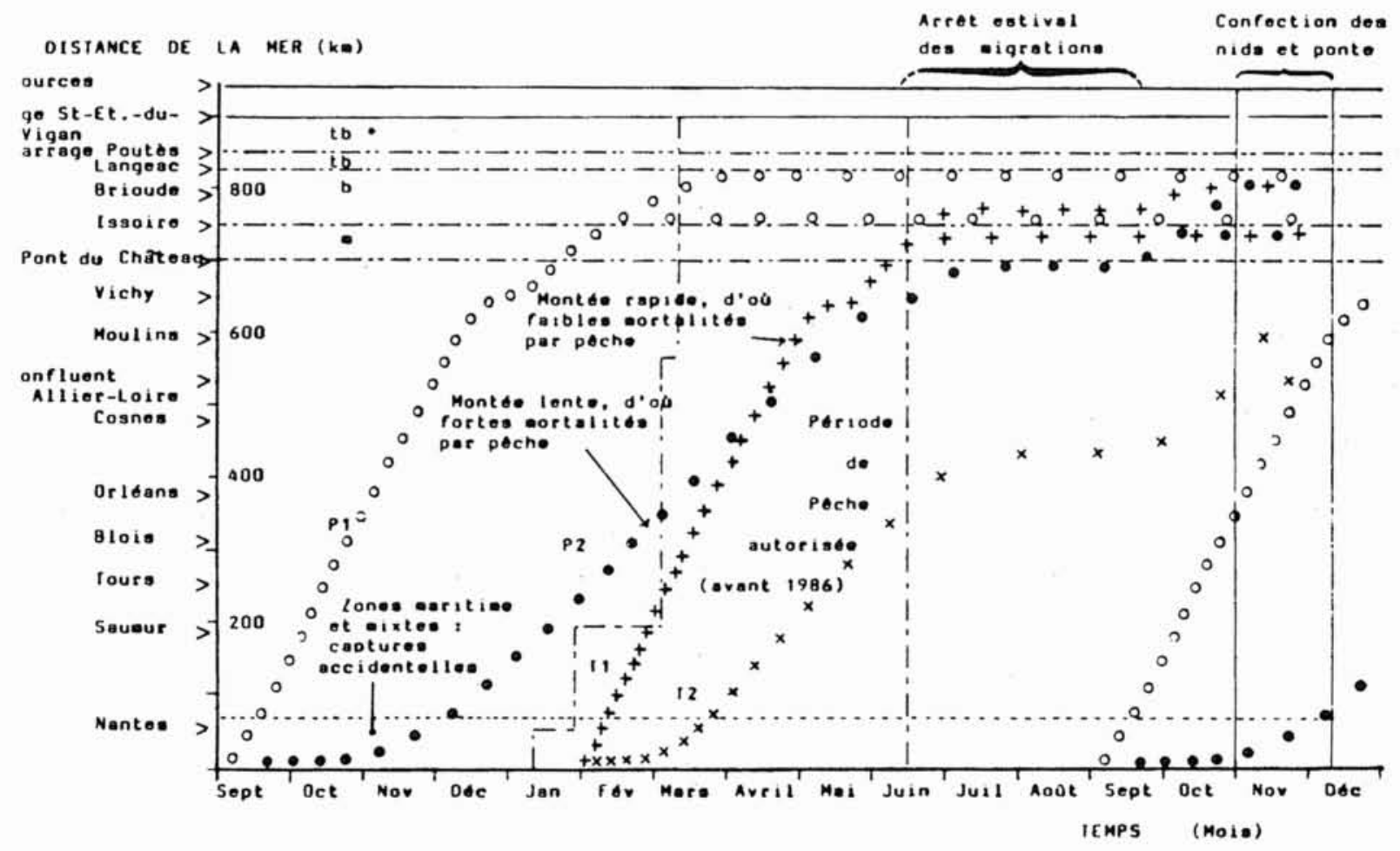

Figure 6 
L'expérience montre que, même équipés de passes à poissons, les obstacles ne sont presque jamais neutralisés, et restent des "obstacles relatifs". Les retards et pertes d'énergie qu'ils provoquent se répercutent tout au long du parcours. Ils favorisent des mortalités excessives par pêche dans certaines zones d'attente. Leurs effets négatifs sur la reproduction sont ainsi cumulatifs, et probablement même multiplicatifs, pouvant conduire insidieusement à l'extinction totale de la population - ce qui s'est produit, en un siècle, sur tous les cours d'eau du Bassin de la Loire sauf un.

Sur les axes fluviaux et affluents où l'on souhaite préserver le Saumon, une politique globale et rigoureuse est donc indispensable, de l'estuaire aux frayères; elle doit s'appliquer à :

1) Equiper tous les obstacles existants de passes à poissons les plus efficaces possibles (pour cela, elles doivent être bien situées, bien construites, bien alimentées et bien entretenues (Larinier, 1983).

2) Mais ne pas se faire d'illusions ếxcessives sur l'efficacité de ces passes (pour certains barrages, perpendiculaires à l'axe de la rivière, ou de grande hauteur, ou à niveau variable, ou avec faible restitution d'eau), le franchissement restera souvent aléatoire. D'où deux corollaires :

3) Tant qu'on n'aura pas la certitude qu'un obstacle est vraiment neutralisé, ce qui est très rare, la pêche du saumon doit être mise en réserve sur une distance importante à l'aval (plusieurs kilomètres sont généralement nécessaires sur l'Allier et sur la Loire).

4) Ne permettre la construction d'aucun nouvel obstacle sur cet axe. Sur ce point, certains des projets récemment présentés par l'E.P.A.L.A. (2) est en contradiction totale avec le programme de restauration du Saumon entrepris sur l'axe Loire-Allier et certains affluents, et qui commence à porter ses fruits.

\section{$* *$}

La qualité et la dimension des frayères confèrent à l'Allier et à certains de ses affluents un potentiel en saumons considérable; nous l'estimons à 15 à 25000 adultes par an, soit cinq à dix fois plus que le cheptel actuel. A ce niveau de population, le poids socio-économique du Saumon sera indiscutable.

Ce potentiel peut devenir réalité à peu de frais dès la prochaine décennie, si l'on maintient simplement les efforts des dix dernières années en matière de règlementation de la pêche et de facilitation de la migration entre la mer et ces frayères.

Ce potentiel restera inemployé et le saumon restera une " espèce menacée " sur ce bassin, si ces efforts se relâchent, et surtout si de nouveaux obstacles sont édifiès sur le parcours.

Dans le choix qui seront faits, il importera de tenir compte non seulement des aspects socio-économiques, mais aussi d'un fait biologique : le patrimoine génétique du saumon Loire-Allier et ses performances migratoires sont tout à fait exceptionnels. Sa disparition de ce bassin serait certainement irréversible.

\section{$* *$}

Nous remercions M.M. Jean-Jacques Demars et Pascal Bomassi pour leur lecture critique du manuscrit et leurs observations.

(2) E.P.A.L.A. : Etablissement public pour laménagement de la Loire et de ses affluents. Le programme actuellement proposé par cet établissement comporte la construction de grands ouvrages pour écrêtement de crues et/ou soutien d'étiage. Il envisage également, sur la Loire en aval du confluent de l'Allier, des barrages à des fins de loisirs, navigation, protection de berges, prises d'eau; I'utilité publique de ces derniers nous paraît contestable; leurs conséquences négatives sur les poissons migrateurs sont par contre certaines.

\section{Bibliographie sommaire}

BACHELIER R., 1964. - Lhistoire du saumon en Loire - Bull. Fr. Piscic., 211, 212, 213-53 p.

THIOULOUZE G., 1979. - Responsabilités des fervents de la pêche sportive sur l'avenir des stocks de rivières telles que l'Allier et le Gave d'Oloron - Saumons, 30, 10-15.

LARINIER M., 1983. - Guide pour la conception des dispositifs de franchissement des barrages pour les poissons migrateurs Bull. Fr. Piscic. ${ }^{\circ}$ spécial, $39 \mathrm{p}$.

CUINAT R., 1980. - Le saumon Loire-Allier: Caractéristiques actuelles et perspectives - Symp. FAO, CECPI, Vichy, avril 1980, dactyl. $18 \mathrm{p}$.

CUINAT R. et P. Bomassı 1985. - Évolution de la situation pour le Saumon du Bassin Loire-Allier de 1979 à 1985 - Colloque Franco-Québecois, Bergerac - Avril 1985, dactyl. 11 P. + figures.

Nota: Les deux dernières études ont été réalisées avec l'aide financière du ministère de l'Environnement (Direction de la nature, Service de la pêche et de l'hydrobiologie). 
M. MORELLO. - Comment peut-on expliquer que des modifications récentes de la réglementation prolongent les périodes de pêche sur l'axe Loire/Allier alors qu'on fait de si gros efforts pour le maintien des stocks de saumons?

M. CUINAT. - Notre Délégation Régionale avait conseillé un retard à la fois de l'ouverture et de la fermeture, en vue de protéger davantage la fraction précoce du contingent. En fait, pour 1986, seul le retard de la fermeture a été décidé, ce qui a abouti en effet à un allongement de la période de pêche.

M. TANE. - La nouvelle réglementation de la pêche introduit une dimension nouvelle dans la gestion des populations piscicoles, attendu que la France ne possède pas encore la connaissance de ses stocks qu'on pourrait attendre d'un pays moderne.

L'année 1986 doit être considérée comme une année transitoire, avec des mesures provisoires à caractère pédagogique. L'exploitation des castillons et la protection des "précoces" semble aller dans ce sens.

A terme, en 1987, les mesures en place concernant les pêcheurs seront :

- la mise en place d'un réseau de collecte de données avec notamment déclaration dans le « carnet de pêche " du nombre des captures par espèce, du lieu de capture.

- des mesures de protection consistant par exemple à l'interdiction de pêcher au niveau des barrages, à l'obligation d'installer des échelles à poissons, d'où les articles 410 et 411 du Code Rural.

Des mesures concernant les barragistes, les producteurs autonomes, et l'Administration, sont aussi nécessaires.

M. THIBAULT. - Jusqu'à maintenant on a considéré le Saumon atlantique comme un ensemble unique sans prendre en compte, en France, les différentes classes d'âge de mer (d'une part un hiver de mer, d'autre part plusieurs hivers de mer) bien que le groupe de travail sur le Saumon de l'Atlantique nord du Conseil International pour l'Exploration de la Mer le recommande depuis 1967. La réglementation modifiée au cours des 20 dernières années sous la pression de certains protecteurs de l'espèce a consisté à réduire progressivement les périodes de pêche (confinées surtout au printemps actuellement) tant en estuaire qu'en eau douce. Ce faisant, l'exploitation a été concentrée sur les poissons de plusieurs hivers de mer alors que ces mêmes protecteurs affirment que ces saumons sont déjà surexploités sur l'aire d'engraissement du Groënland. Les poissons d'un hiver de mer (les castillons) qui ne vont pas au Groënland sont ainsi quasi-inexploités par la pêche à la ligne.

En nous basant sur les résultats obtenus au laboratoire, une modification de la période de pêche a été proposée, sans succès, dès 1977 pour la Bretagne afin de permettre l'accès à la composante castillon de la ressource. L'objectif était de décaler la période de pêche en repoussant la fermeture jusqu'en novembre. De telles modifications ont été réalisées dans le sud ouest de l'Angleterre, région très comparable à la Bretagne. Une telle mesure doit s'accompagner d'un suivi scientifique afin de connaitre son incidence sur le stock.

J'insiste à nouveau sur la présence des fluctuations annuelles dans les captures qui ne reflètent pas obligatoirement les fluctuations d'abondance du stock. Il ne faut pas oublier enfin l'influence essentielle de l'environnement marin d'une part sur l'importance du stock (par exemple les pics des captures de saumon en Suède en 1880-90 et 1940-50) d'autre part sur l'importance relative des différentes classes d'âge de mer (diminution des saumons de plusieurs hivers de mer depuis un demi-siècle en Ecosse).

M. LEGRAND. - Pendant sa migration le saumon ne s'alimente pas, a-t-il été dit. Comment expliquer ses captures à la ligne?

M. CUinat. - Certains parlent de réflexes que le poisson aurait conservé de ses comportements alimentaires, soit acquis en mer (mais on peut objecter que le type de proies qu'il peut attraper en rivière n'est pas comparable), soit acquis pendant sa phase juvénile. Il pourrait aussi s'agir d'agressivité en liaison avec un réflexe de défense. Cette question mériterait d'être discutée plus longuement, mais elle ne constitue pas l'objet de la séance. 